\title{
Modified functional ocularimpression of post-enucleation socket: A case report
}

\author{
${ }^{1}$ Fauzan Arif, ${ }^{2}$ Ariyani, ${ }^{2}$ Haslinda Z. Tamin \\ ${ }^{1}$ Postgraduate Program in Prosthodontics \\ ${ }^{2}$ Department of Prosthodontics \\ Faculty of Dentistry, Universitas Sumatera Utara \\ Medan, Indonesia \\ Corresponding author: Fauzan Arif, E-mail: Fauzandentist@gmail.com
}

\begin{abstract}
Introduction:The disfigurement associated with the loss ofan eye can cause significant physical and psychological problems. Therefore, a good ocular prosthesis is needed to restore the patient's quality of life. One of the factors that determine the quality of an ocular prosthesis is accurately impression. In the case of enucleation where the eyes socket has a soft and movable tissue bad will result inaccurate impression. The difficulty of post enuclation impression in such case is the compression of the tissue eyes socket. This case report will discuss the modification in functional impression technique using a modified custom tray. Case: A 39-year-old male patient came to Dental Hospital Universitas Sumatera Utara with chief complaint an unaesthetic and loose left stock eye that had been used for 10 years. Clinical examination shows moderate depth eye socket with soft and movable tissue. Management: The functional impression technique was modified using a self-curing clear acrylic sclera without handle as a custom tray with $2 \mathrm{~mm}$ reduction on the intaglio surface and light body polyvinyl siloxane as impression material. Discussion: The aim of this modification is to avoid the tendency of the eye socket to compress during impression taking and to achieve a natural contour of the eyelid convexity because of the absence of the tray handle so the patient can close their eyes. Conclusion: This technique produces an ocular prosthesis that has a good retention with excellent aesthetic result.
\end{abstract}

Keyword: enucleation, modified functional impression, ocular prosthesis

This title has been presented in The 12th Biennial Congress of Asian Academy of Prosthodontics, 21 August 2021

\section{INTRODUCTION}

The special sensory organs play significant role in daily lives. The most tragic and most commonly occurring loss of one of these sensory organs is that of eye. The disfigurement associated with the loss of an eye can cause significant physical and psychological problems. Thus, the replacement of the lost eye is necessary to promote physical and psychological healing for the patient and to improve social appearance. ${ }^{1,2}$

Loss of the eyeball can be caused by congenital anomalies, trauma and tumors. Surgical procedures in making ocular prosthesis are classified into 3 categories: evisceration, enucleation and exenteration. Evisceration is involved the removal of the contents of the globe leaving in place the sclera and sometimes the cornea. The prosthesis best suited for the evisceration defect is the custom cover shell or the scleral cover shell prosthesis. Enucleation is the removal of the entire globe after the extra ocular muscles and the optic nerve have been transected. The prosthesis best suited for the defectis conventional or implant supported ocular prosthesis. Exenteration is the removal of the entire contents of the orbit (entire eye and surrounding structures). This procedure is usually performed due to some form of malignant disease. ${ }^{1-3}$
Rehabilitation forthe loss of eye is divided into two types: implants and ocular prostheses. Ocular prostheses are divided into stock eyes and custom ocular prostheses. In the case of enucleation if there is sufficient space for ocular prosthesis, the latestadvance treatment is implant because it can provide retention and good appearance. Although implants are the best option, these treatments are expensive and cannot be afforded by everyone. Another best treatmentalternative is a custom eye prosthesis because it can duplicate the anatomy of the eye defect properlyand affordable., ${ }^{4,5}$

One of the successes in making ocular prosthesis is the accurate impression of the anatomical area of the eye defect. Numerous impressiontechniques have been described in the literature. Smith uses an existing custom eye as impression tray using the relain technique. The advantage of this technique compared to other techniques such as stock oculareyes is that the eye contour is already formed in both the intaglio and anterior regions so it can produce accurate eye impressions. ${ }^{6}$

\section{CASE}

A39-year-old male patient came to dental hospital Universitas Sumatera Utara with chief complaint an unaesthetic and loose leftstock eye that 
had been used for 10 years. The patient experierienced sharp trauma to his left eye when he was 29 years old and the entire eyeball was removed at the Zainal Abidin General Hospital in Banda Aceh and after recovering the patient used stock eyes.

Onclinical examination of the intraocular tissue (Fig.1), the orbital socket mucosa was in good health, the depth of the eye socket was deep, especially in the superior palpebral area, there was ptosis, lower eyelid laxity, and excessive mucus (the patient rarely opened and cleaned the stock eyes).

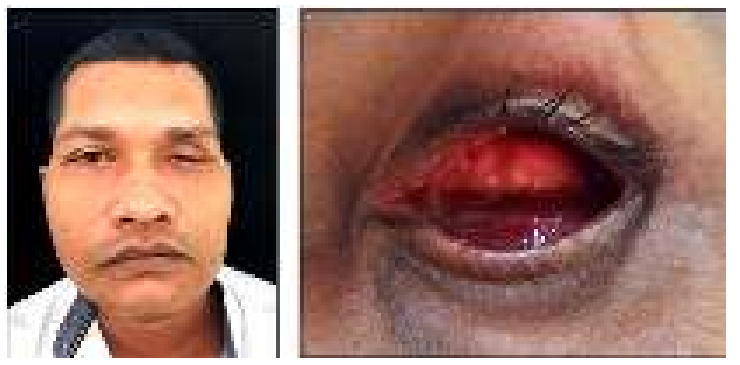

Figure 1 Extra and intra ocular examination

\section{MANAGEMENT}

The primaryimpression is done with a tray that made using self-cured acrylic which is shaped in such a way using the thumb and adjusted to the contour and size of the eye socket. After the acrylic setting time is complete, then a handle tray and holes are made for retention. The tray is smoothed and polished (Fig. 2).

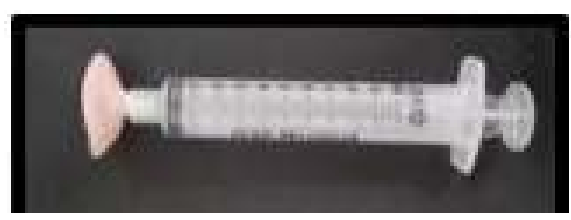

Figure 2 Primary tray

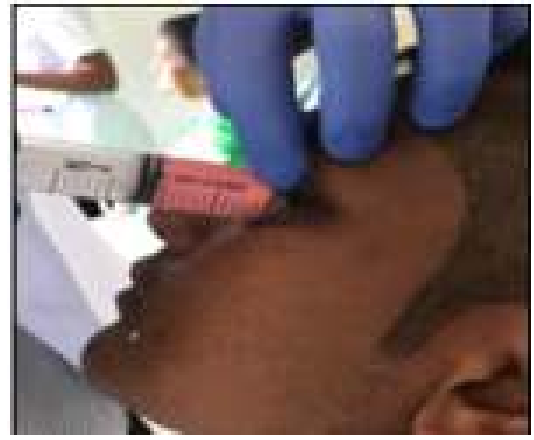

Figure 3 primary impression

The tray is tested on the patient. The hydrocolloid irreversible impression material (Alginate) is inserted into the syringe, the tray is placed into the socketand then injected. The patientwas seat- ed in an upright position and instructed to move the eyeball to the right, left, up and down without moving the head to record the proper depth and width of the socket (Fig.3). After the material set, the tray is removed and the remaining impression material is cleaned from the socket.

The surface of the intaglio tray impression is planted in a small mold containing half of the dental stone to the anterior posterior edge of the mold and allowed to be harden. After the dental stone hardens, makekey holes in the medial and lateral canthus. Apply vaseline on the upper surface and then pour back the dental stone as the antagonist and allowit to harden, make a key hole on the top surface of the antagonist model, a hole is made as an entrance for the melted wax (Fig.4).
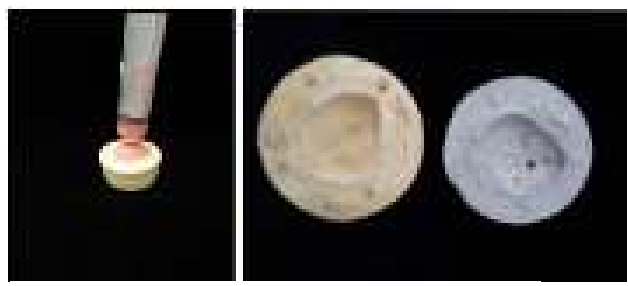

Figure 4 Mold making

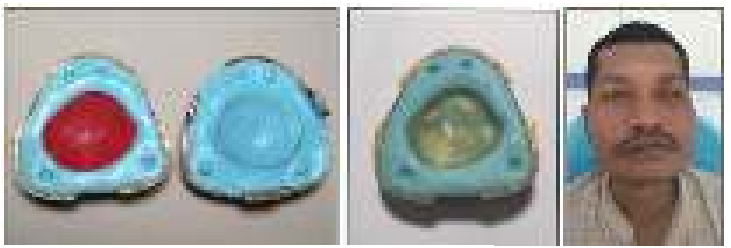

Figure 5 Clear acrilyc sclera

Making scleral wax; the surface of the mold is moistened with water and then the molten wax is poured into the mold. Then wax pattern is shaped by the natural convexity of the eye with the highest part of the convexity located in the pupillary area. Trial on patients to get the shape of the eyeball that best fit the natural eye. After everything have been matched, the surface of the wax pattern is smoothed.

To make temporary sclera with clear acrylic material, place half of the sclera wax in the lower flask which has been filled with putty impression material, wait till set and make a key hole then cup upper flask and fill it with putty impression material then close and press until the material sets. Remove the sclera wax and fill it with clear acrylic material then press and wait for it to set. Remove excess on the sclera and polish and perform a trial on the patient. Note the convexity and retention of the transient scelera (Fig.5).

Modified functional ocular impression used clear acrylic scelera. After try in the acrylic sclera 
on the patient, the intaglio surface of the sclera is reduced by $2 \mathrm{~mm}$ then apply adhesive tray material and then using light body impression material on the acrylicsclera and insert it back into the eye socket. Instruct the patient to perform physiological eye movements such as moving the eyes to the right, left, up, down, closing and opening the eyes. With this technique, an accurate impression of the anatomical part of the defect area and natural eye convexity is obtained (Fig.6).

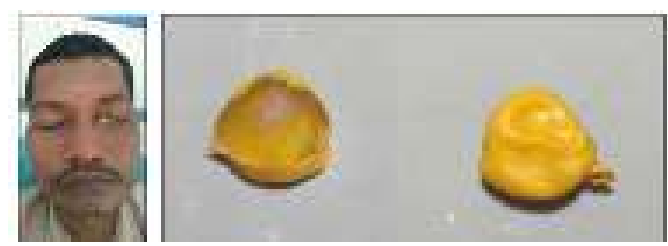

Figure 6 Functional ocular impression

Then the sclera was planted in mold the same as before to get a definitive mold for making sclera wax. The final sclera wax is put to try on the patient. The patient's eye convexity, eye movement and retention was evaluated.
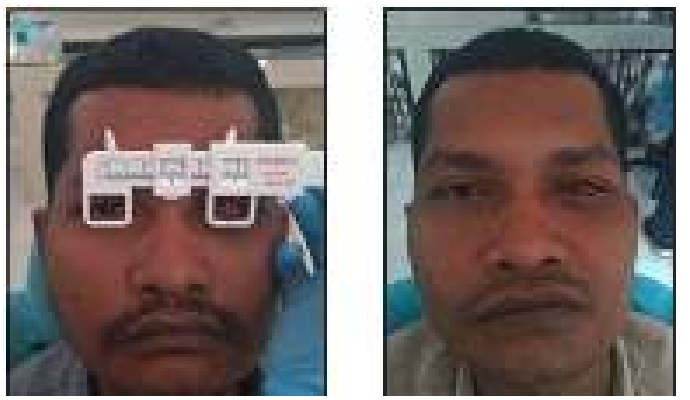

Figure 7 Pupil midpoint determination

Determination of the position of the midpoint of the pupil using the interpupillary distance (IPD) ruler(Fig.7). First, determine the midpointbetween the medial canthus of the left and right eyes as the guide point for the IPD ruler, marked with a marker, then the position of the midpoint of the right pupil is adjusted to the position of the midpoint of the pupil of the left eye. In this patient, the distance from the IPD ruler guide point to the midpoint of the original pupil was $3.1 \mathrm{~cm}$. The diameter of the iris was determined $(11.5 \mathrm{~mm})$, then the circle is colored with a marker on the entire iris.

The iris coloring uses a paper iris disk technique with modifications (the paper material is replaced with a black disk of the doll's eye) using acrylic paint with a mixture of brown and black colors adjusted to the color of the iris of the other eye, and then planted in the mold and then packaged using heat cure clear acrylic to get the iris button. Then the iris button is inserted in the scelera waxaccording to the midpoint obtained and a trial is performed on the patient (Fig.8)
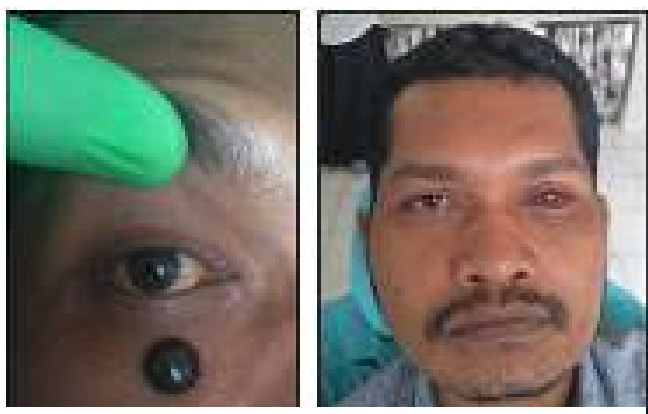

Figure 8 Trial sclera and iris

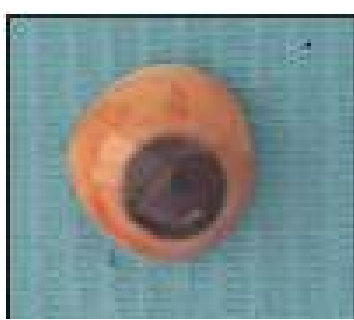

Figure 9 The ocular prosthesis

Making the sclera of the eye by replanting the sclera wax that already has the iris button back into antagonist of the cuvet (in the iris button, plus the acrylic rod, so that the iris button does not rotate when boiling) then cup the top of the flask and fill it with type IV dental stone, close and press. Evaluate convexity, eye movement and retention of the scelera. When it has been adequate, the anterior surface can be reduced by $1 \mathrm{~mm}$ for clear acrylic and blood vessels. Then, the sclera was flasked and packed using white acrylic (Fig. 9)

The final prosthesis was fitted with full attention to appearance, comfortable and function (Fig. 10). The patient was given instructions on how to put on and remove the ocular prosthesis, care at home should be done with care and clean hands, the ocular prosthesis was removed at night and immersed in water. antibacterial solution. Recall were performed on days 1, 3, 7 post-insertion.

\section{DISCUSSION}

The disfigurement associated with the loss of an eye can cause significant physical and psychological problems. The loss of eye has a serious impact notonly for patient but also on family membersand the society. Most patient experience severe stress, especially when adapting to functional disability caused by eye loss and reactions from society. Therefore, restore the patient's quality of life depends on a good eye prosthesis. ${ }^{1,2}$

The maingoals in performing maxillofacial rehabilitation are 1) restore aesthetics and cosmetics, 
2) restore function (which is still acceptable), 3) protecting existing tissue, 4 ) the prosthesis provivides therapeutic or healing effects, and5) the prothesis provide psychological effects. ${ }^{2}$

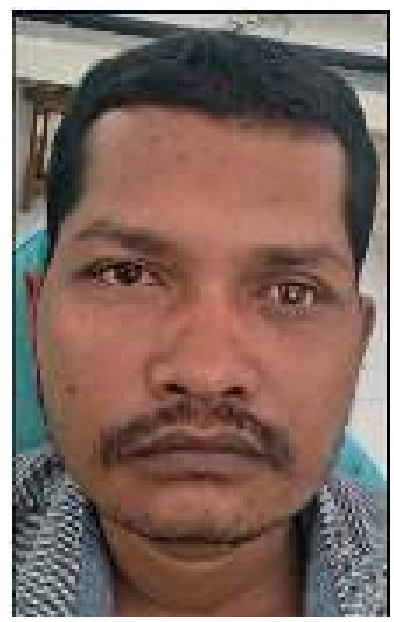

Figure 10 The patient used his ocular prosthesis

At every stage of making an ocular prosthesis presented in this case, all of the objectives have been fulfilled. Protheses were made well and planned by maintaining orientation of functional move of the patient eyes. With the development of new materials and modifications of ocular impression techniques, the eye sockets can be recorded in detail, resulting in acrylicocularprostheses that can be made with accurate fit and aesthetics.

A retentive ocular prosthesis is obtained from a detailed impression that can record anatomical of eye defects and eye convexity accurately so it can result ocular prostheses that are both functionally and aesthetically pleasing. In the enucleation procedure, afterhealing the eye socket will be found a soft tissue and easy to move. This situation will make the impression difficult because there is a tendency for this tissue to be compressed so that it will produce inaccurate printing. . $^{1,2,7}$

Smith in 1995 described the relaining procedure on existing ophthalmic prostheses using Korectawaxno. 4 and performing functional impressions and concluded that the functional reline im- pression technique resulted in retentive prosthesis adaptation and improved patient appearance and psychology. ${ }^{6}$ With the development of impression materials such as flexible impression materials, it will be advantageous when the defect extends beyond the orbital region and can overcome with moving mucosal tissue. According to Turne, "light body printing materials have good biocompatibility and good dimensional stability so that they are very accurate in eye printing compared to alginate."1,7

Based on the impression technique described by Smith and the latest developments in printing materials, this paper modifies the impression technique with a functional method using self-curing clear acrylic sclera which acts as a tray with a $2 \mathrm{~mm}$ reduction in the intaglio portion and using light body impression material as an impression material.

Compared to stock eyes, custom eye prostheses provide the best compatibility with ocular tissues because provide good adaptation, thereby reducing irritation, infection or fluid accumulation at the prosthesis-tissue interface. Due to the tight adaptation to the underlying tissue, it can result in bettermobility of the prosthesis, uniform pressure distribution and reduced incidence of conjunctival abrasion and ulceration compared to the stock prostheses. Control overtheorientation, color, contour, and size of different parts of the eye, resulting in facial symmetry and superior aesthetics. ${ }^{3,9,10}$

It is concluded that the functional imprinting technique that has been modified in this case allows the eye prosthesis to move simultaneously following the patient's original eye without being separated by this movement due to the close contact between the eye prosthesis and the eye socket thereby improving the appearance of the eye. Because there is no handle of tray on the anterior aspectensures that the outer eye contour resembles that of the adjacent normal eye. This results in excellent aesthetics and functionality. This technique has givengood results from both the patient.

\section{REFERENCES}

1. Ravi Y, Srinivas K, Singht M. A new procedure for the fabrication of custom ocular prosthesis - a case report. Dent J Adv Studies 2013.

2. Jethwani J, Jethwani GS, Verma AK. Functional impression technique for an ocular prosthesis. J India Prosthodont Soc 2012:12(1):55-8.

3. Da Costa GC. Ocular prosthesis incorporating IPS e-max press scleral veneer and a literature review on nonintegrated ocular prosthesis. Int J Ophthalmol 2017:10(1):148-56.

4. Tripuraneni SC. An innovative impression technique for fabrication of a custom-made ocular prosthesis. Indian J Ophthalmol 2015:63(6): 545-7.

5. Baslas V. Basic principles of rehabilitation for lost eye: A dentist's perspectives. J Sci Soc 2015:42(2):99-102.

6. Smith RA. Relaining an ocular prosthesis: a case report. J Prosthodont 1995;4(3);160-3 
7. Turner JM, Purslow C, Murphy PJ. Ocular impression-taking-which material is best? Contact Lens Association of Ophthalmologists 2018:1-6.

8. Bali N, Dhall RS, Singh N. Various steps involved in fabrication of an ocular prosthesis: a case report. Int J Dent Med Res 2015:1(5): 93-6.

9. Manoj SS. Modified impression technique for fabrication of a custom-made ocular prosthesis. Anaplastol 2014:3(2):129-30.

10. Shetty PP. A iris positioning device and centering approach: A technique. J Prosthet $2017: 1-3$. 